\title{
Identification, Pyramid and Candidate Genes of QTLs for Associated Traits Based on a Dense Erect Panicle Rice CSSL-Z749 and Five SSSLs, Three DSSLs and One TSSL
}

\section{Dachuan Wang}

Southwest University

Kai Zhou

Southwest University

Siqian Xiang

Southwest University

Qiuli Zhang

Southwest University

Ruxiang Li

Southwest University

Miaomiao Li

Southwest University

Peixuan Liang

Southwest University

Naz Farkhanda

Southwest University

Guanghua He

Southwest University

Yinghua Ling

Southwest University

Fangming Zhao ( $\sim$ zhaofangming2004@163.com)

Southwest University https://orcid.org/0000-0003-2781-0452

Original article

Keywords: Rice, Chromosome segment substitution lines, Panicle related traits, QTL, Fine mapping, Additive and epistatic effects

Posted Date: January 20th, 2021 
DOI: https://doi.org/10.21203/rs.3.rs-148666/v1

License: (c) (1) This work is licensed under a Creative Commons Attribution 4.0 International License. Read Full License 


\section{Abstract \\ Background}

Seed-set density is an important agronomic trait in rice. However, its genetic mechanism is complex. Chromosome segment substitution lines (CSSLs) are ideal materials for studying complex traits.

\section{Results}

A rice $C S S L, Z 749$, with a dense and erect panicle phenotype, was identified among progeny of the recipient parent Nipponbare and the donor parent Xihui 18. Z749 carried seven substitution segments (average length $2.16 \mathrm{Mb}$ ). Compared with Nipponbare, Z749 showed significant increases in the numbers of primary (NPB) and secondary branches (NSB), number of spikelets (SPP) and grains per panicle (GPP), seed-set density (SSD), and decrease in panicle length $(\mathrm{PL})$. A secondary $\mathrm{F}_{2}$ population derived from a cross between Nipponbare and Z749 was used to map quantitative trait loci (QTLs) for associated traits. Fifteen QTLs distributed on chromosomes 5, 7, 8, and 10 were detected. The QTL $q P L 7$ might be an allele of OSFAD8 and the remaining 14 QTLs (e.g., qSSD5 and qSSD10 etc.) might be novel. Fourteen QTLs were verified using five single-segment substitution lines (SSSLs). The seed-set density of Z749 was controlled predominantly by one major QTL ( $q S S D 10$ ) and two minor QTLs ( $q S S D 5$ and $q S S D 8$ ). The QTLs qSSD10, $q S S D 5$, and $q S S D 8$ were fine-mapped to intervals of $1.05,1.46$, and $1.53 \mathrm{Mb}$ on chromosomes 10,5 , and 8, respectively. Analysis of QTL additive effects indicated that $q S S D 5, q S S D 8$, and $q S S D 10$ from Xihui18 increased seed-set density of $Z 749$ by $14.1,11.38$, and 5.11 spikelets per $10 \mathrm{~cm}$ panicle, respectively. Analysis of QTL epistatic effects revealed that pyramiding of $q S S D 5$ and $q S S D 8, q S S D 5$ and $q S S D 10$, $q S S D 8$ and $q S S D 10$, and $q S S D 5, q S S D 8$ and $q S S D 10$ produced novel genotypes with increased seed-set density.

\section{Conclusions}

Inheritance of seed-set density in Z749 was controlled predominantly by one major QTL ( $q S S D 10$ ) and two minor QTLs ( $q S S D 5$ and $q S S D 8$ ). Then, they were fine-mapped to intervals of $1.05,1.46$, and $1.53 \mathrm{Mb}$ on chromosomes 10, 5, 8, respectively. Two MAPK genes (OSMPK9 and OSMPK17) and one gene (candidate gene 6) involved in auxin metabolism might be candidate genes for qSSD5, and OSSAUR32 might be the candidate gene for $q S S D 8$. Pyramiding of $q S S D 5, q S S D 8$, and $q S S D 10$ enhanced seed-set density.

\section{Background}

Rice (Oryza sativa L.) is an important cereal crop and a staple food for more than $50 \%$ of the global population (Gathala et al. 2011). Improvement of the yield continues to be a crucial focus in rice breeding. Panicle architecture is an important agronomic trait that affects seed-set density and is strongly 
associated with grain yield. Assuming no changes to other components, an increase in seed-set density can improve rice yield. However, the genetic mechanism for seed-set density is complex and controlled by multiple genes.

In recent decades, a number of genes have been shown to affect the architecture of the rice panicle. Some of these genes are involved in the hormone signaling pathways of phytohormones, such as cytokinins, auxin, and brassinolactone. Cytokinins regulate the size of reproductive meristematic tissue, and auxin is involved in the formation of meristematic tissue, thereby affecting panicle development (Wang et al. 2018). Genes associated with the cytokinin metabolism pathway include Gn1A (GRAIN NUMBER 1A; OSCKX2), DST (DROUGHT AND SALT TOLERANCE), SMG1 (SMALL GRAIN 1), GSN1 (GRAIN SIZE AND NUMBER 1), OSER1 (ERECTA 1), DEP1 (DENSE AND ERECT PANICLE 1) and LP(LARGER PANICLE). The gene Gn1A encodes an enzyme that catalyzes the degradation of active cytokinins. Reduced Gn1A expression may lead to accumulation of cytokinins in the inflorescence meristems, thereby increasing the number of spikelets and leading ultimately to denser panicles (Ashikari et al. 2005). The genes DST, SMG1, GSN1, OsER1, DEP1 and LP can affect the cytokinin content in reproductive meristems by direct or indirect upregulating of the transcription of $G n 1 A$, and can therefore affect panicle development (Zhang and Yuan 2014; Li et al. 2013; Duan et al. 2014; Guo et al. 2018; Guo et al. 2020; Huang et al. 2009; Liu et al. 2018; Li et al. 2011; Piao et al. 2009). Genes associated with the auxin metabolism pathway include PAY1 (PLANT ARCHITECTURE AND YIELD 1), LAX1 (LAX PANICLE 1), SPA (SMALL PANICLE) and ASP1 (ABERRANT SPIKELET AND PANICLE1). PAY1 may improve the architecture of the plant panicle by altering distribution of endogenous indole-3-acetic acid and affecting polar auxin transport activity (Zhao et al. 2015). $L A X 1, S P A$, and ASP1 are indicated to play crucial roles in auxinmediated panicle development but their mode of action is not clear (Zhang and Yuan 2014, Keishi et al. 2003; Shen et al. 2010; Yoshida et al. 2012). DWARF 11 (D11, CPB1) is involved in the brassinolide biosynthetic pathway. The $c p b 1$ mutant shows phenotypes of increased BR-sensitivity, clustered primary branches of the panicle, and smaller seeds (Wu et al. 2016). However, it remains unclear how brassinolide affects panicle development. In addition, certain genes regulate rice panicle development through other pathways, such as DEP2, DEP3, OSMFT1 (MOTHER OF FT AND TFL 1) and FUWA. (Li et al. 2010; Liu et al. 2015; Qiao et al. 2011; Song et al. 2018; Chen et al. 2015). Although many genes associated with panicle architecture have been cloned, they are insufficient to explain the complexity of the genetic mechanism. Therefore, it is necessary to identify additional genes that influence the dense panicle architecture of rice.

Chromosome segment substitution lines (CSSLs) are ideal materials to use for genetic research of complex traits and genetic resources for crop improvement (Zhang et al. 2019; Balakrishnan et al. 2019). In the present study, we identified a rice CSSL, Z749, with a dense and erect panicle phenotype, derived from 'Nipponbare' as the recipient parent and 'Xihui 18' as the donor parent. We characterized Z749 genetically and mapped quantitative trait loci (QTLs) for associated traits using a secondary $F_{2}$ population derived from a cross between Nipponbare and Z749. In addition, we developed single-segment (SSSLs), double-segment (DSSLs), and triple-segment substitution lines (TSSLs) for each QTL in the $F_{3}$ generation using marker-assisted selection (MAS). We verified the accuracy of QTL mapping using 
SSSLs. We conducted analysis of the additive and epistatic effects of QTLs on seed-set density and assessed the effect of QTL pyramiding using DSSLs and TSSLs. In addition, the QTLs qSSD5, qSSD8, and qSSD10 were fine-mapped and candidate genes were analysed.

\section{Results}

\section{Identification of substitution segments in Z749}

Identification of the substitution segments and detection of the genetic background purity were performed on 10 individuals of the Z749 lines using all simple sequence repeat (SSR) markers located on the seven substitution segments of Z749 and 24 SSR markers, which were in turn located outside the substitution segments. The results showed that Z749 was homozygous and no additional residual segment was detected. Seven chromosomal substitution segments derived from Xihui 18 were located on chromosomes 5, 7, 8, 10, and 11. Chromosomes 7 and 10 each contained two substitution segments. The total substitution length was $15.09 \mathrm{Mb}$, the longest substitution was $4.09 \mathrm{Mb}$, the shortest substitution was $0.73 \mathrm{Mb}$, and the mean substitution length was $2.16 \mathrm{Mb}$ (Fig. 1).

\section{Phenotypes of Cssl-z749}

Compared with Nipponbare, CSSL-Z749 showed significant increase in number of grains per panicle, number of spikelets per panicle, number of primary branches, number of secondary branches, and seedset density, which were increased by $15.51 \%, 23.92 \%, 29.57 \%, 23.65 \%$, and $59.42 \%$, respectively. The seedset density of Z749 was 82.34 spikelets per $10 \mathrm{~cm}$ panicle length, whereas that of Nipponbare was 51.65 (Fig. 2A, B, C, D, F, G, H). The panicle length of Z749 was decreased significantly compared with that of Nipponbare (Fig. 2B, E). Therefore, the increased seed-set density of Z749 was caused predominantly by a reduction in the panicle length and increases in the numbers of branches and spikelets per panicle. For other agronomic traits, such as heading date and 1000-grain weight (Fig. 2A), no significant differences were observed between Z749 and Nipponbare.

\section{QTL mapping for seed-set density related traits in secondary $F_{2}$ population of Nipponbare/Z749}

The substitution segments of Z749 carried 15 QTLs for traits associated with seed-set density. The percentage phenotypic variation explained by individual QTLs ranged from 4.68-21.01\%. Seven QTLs were major and contributed to panicle length ( $q P L 7$ and $q P L 10$ ), number of primary branches ( $q N P B 7)$, number of secondary branches ( $q N S B 7$ and $q N S B 10$ ), number of grains per panicle ( $q S P P 10$ ), and seedset density (qSSD10). The remaining eight QTLs showed minor effects (Table 1, Fig. 1). 
Table 1

QTL for rice seed-set density related traits detected in Z749

\begin{tabular}{|c|c|c|c|c|c|c|c|}
\hline Trait & QTL & Chr. & $\begin{array}{l}\text { Linked } \\
\text { marker }\end{array}$ & $\begin{array}{l}\text { Additive } \\
\text { effect }\end{array}$ & $\begin{array}{l}\text { Var. } \\
(\%)\end{array}$ & $\begin{array}{l}P \\
\text { value }\end{array}$ & $\begin{array}{l}\text { Possible } \\
\text { alleles }\end{array}$ \\
\hline \multirow[t]{2}{*}{ Panicle length $(\mathrm{cm})$} & $q P L 7$ & 7 & RM2715 & 1.47 & 13.98 & 0.0020 & $\begin{array}{l}\text { OsFAD8 (Nai } \\
\text { et al. 2009) }\end{array}$ \\
\hline & qPL 10 & 10 & RM8207 & -2.09 & 21.01 & 0.0192 & - \\
\hline \multirow{3}{*}{$\begin{array}{l}\text { Number of primary } \\
\text { branch }\end{array}$} & $q N P B 7$ & 7 & RM2715 & 0.65 & 13.54 & 0.0032 & - \\
\hline & $q N P B 8$ & 8 & RM1235 & 0.59 & 5.69 & 0.0018 & $\begin{array}{l}\text { OsREL2 } \\
\text { (Kwon et al. } \\
2012)\end{array}$ \\
\hline & qNPB10 & 10 & RM8207 & 0.56 & 9.16 & 0.0103 & - \\
\hline \multirow{4}{*}{$\begin{array}{l}\text { Number of secondary } \\
\text { branch }\end{array}$} & $q N S B 5$ & 5 & RM3170 & 1.79 & 5.46 & 0.0157 & - \\
\hline & $q N S B 7$ & 7 & RM2715 & 3.13 & 13.48 & 0.0038 & - \\
\hline & $q N S B 8$ & 8 & RM1235 & 3.26 & 7.38 & 0.0081 & $\begin{array}{l}\text { OsREL2 } \\
\text { (Kwon et al. } \\
2012 \text { ) }\end{array}$ \\
\hline & qNSB10 & 10 & RM8207 & 2.93 & 12.07 & 0.0059 & - \\
\hline \multirow[t]{3}{*}{$\begin{array}{l}\text { Spikelet number per } \\
\text { panicle }\end{array}$} & qSPP5 & 5 & RM3170 & 7.24 & 5.67 & 0.0136 & $\begin{array}{l}\text { OsPUP7 (Qi } \\
\text { and Xiong } \\
\text { 2013) }\end{array}$ \\
\hline & qSPP8 & 8 & RM1235 & 10.30 & 4.68 & 0.0278 & - \\
\hline & qSPP10 & 10 & RM8207 & 12.07 & 13.01 & 0.0031 & - \\
\hline Seed-set density & qSSD5 & 5 & RM3170 & 3.30 & 6.15 & 0.0104 & $\begin{array}{l}\text { OsPUP7 (Qi } \\
\text { and Xiong } \\
2013 \text { ) }\end{array}$ \\
\hline $\begin{array}{l}\text { (spikelets per } 10 \mathrm{~cm} \\
\text { panicle }\end{array}$ & qSSD8 & 8 & RM1235 & 5.68 & 7.44 & 0.0081 & - \\
\hline length) & qSSD10 & 10 & RM8207 & 6.88 & 20.20 & 0.0002 & - \\
\hline
\end{tabular}

The panicle length of Z749 was controlled by one major QTL ( $q P L 7)$ that increased panicle length by $1.47 \mathrm{~cm}$ and one major QTL ( $q P L 10$ ) that reduced panicle length by $2.09 \mathrm{~cm}$. The number of spikelets per panicle of Z749 was controlled by one major QTL ( $9 S P P 10$ ) and two minor QTLs ( $q S P P 5$ and qSPP8) that increased the trait. The seed-set density of Z749 was controlled by one major QTL ( $($ SSD10) and two minor QTLs ( $q S S D 5$ and $q S S D 8$ ) that increased the trait. 
Five QTLs might be alleles of previously reported genes (Nair et al. 2009; Kwon et al. 2012; Qi and Xiong 2013) (Table 1).

With regard to the possible alleles OSFAD8 (FATTY ACID DESATURASE; Os07g0693800), OsREL2 (ROLLED AND ERECT LEAF 2; Os08g0162100), and OSPUP7 (PURINE PERMEASE 7; Os05g0556800) in Table 1, we performed a comparative DNA sequencing analysis between Nipponbare and Z749. However, no differences in the OSREL2, and OSPUP7 sequences between Nipponbare and Z749 were observed, which indicates that these were not the candidate genes for $q N P B 8$, qNSB8, qSPP5, and $q S S D 5$. For OSFAD8, a single nucleotide polymorphism (SNP) difference in the DNA sequence between Nipponbare and Z749 was detected. The 85th base (the 85th base of the first exon) was mutated from $G$ in Nipponbare to $A$ in Z749. This transition caused a change of amino acid from Glycine to Arginine (Fig. 3). Thus, $q P L 7$ might be an allele of OSFAD8.

\section{Verification of QTLs using SSSLs and analysis of epistatic effects of QTLs for seed-set density using DSSLS and TSSL}

On the basis of the QTL mapping, we developed five SSSLs (S1, S2, S3, S4, and S5), three DSSLs (D1, D2, and D3), and one TSSL (T1) in the $F_{3}$ population by MAS (Fig. 4). The substitution segments of $S 1$ and S2 were located on chromosome 10, with overlapping substitution segments, but the length of the substitution segment of $\mathrm{S} 2$ was shorter than that of S1. Four QTLs were validated by S1, namely qNSB10, qSPP10, qSSD10, and qPL10, whereas one minor QTL ( $q N P B 10$ ) was not detected, whose percentage contribution was $9.16 \%$ in 2018. These results show that some minor QTLs might be influenced strongly by the environment. These QTLs were not detected in S2, which indicates that the QTLs were located in the substitution interval of RM5348-RM8207-RM3311 (Figs. 4, 5). The substitution segment of S3 was located on chromosome 5 on which four QTLs ( $q N S B 5, q P L 5$, qSPP5, and qSSD5) were detected. The S4 substitution segment was located on chromosome 8 on which five QTLs were identified, namely qNPB8, qNSB8, $9 S P P 8, q S S D 8$ and $q P L 8$. The substitution segment of S5 was located on chromosome 7 and four QTLs were identified, which consisted of $q N P B 7$, qNSB7, qSPP7 and $q P L 7$ (Fig. 4). The QTLs $q P L 5, q P L 8$, and $q S P P 7$ were detected in the corresponding SSSLs S3, S4, and S5, respectively. Although not detected in the secondary $\mathrm{F}_{2}$ segregation population of Nipponbare/Z749, the percentage contributions of $q P L 5$, $q P L 8$, and $q S P P 7$ were $11.29 \%, 11.95 \%$, and $5.50 \%$ in 2020 , which suggests that the SSSL showed a higher QTL detection efficiency (Figs. 4, 5). Thus, 14 of the 15 QTLs identified in 2018 were verified by SSSLs in 2020, thus indicating that the QTLs were mapped accurately.

The DSSL D1 carried substitution segments of chromosomes 5 and 8. The substitution segment of chromosome 5 contained $q S S D 5$, the additive effect of which was 14.10 spikelets per $10 \mathrm{~cm}$ panicle length. The substitution fragment of chromosome 8 contained $q S S D 8$, whose additive effect was 11.38 spikelets per $10 \mathrm{~cm}$ panicle length. The epistatic effect between $q S S D 5$ and $q S S D 8$ was -5.26 . Thus, the pyramiding of $q S S D 5$ and $q S S D 8$ produced a genetic effect of 20.22 spikelets per $10 \mathrm{~cm}$ panicle length and therefore yielded denser panicles than those of S3 and S4. The DSSL D2 carried substitution segments of chromosomes 5 and 10. The epistatic effect between qSSD5 $(a=14.10)$ and $q S S D 10(a=$ 
5.11) produced was -4.42 . Thus, pyramiding of $q S S D 5$ and $q S S D 10$ produced a genetic effect of 14.79 spikelets per $10 \mathrm{~cm}$ panicle length in D2, which was larger than the additive effect of S3 (containing qSSD5) and S1 (containing qSSD10). The DSSL D3 carried substitution segments of chromosomes 8 and 10. Pyramiding of $q S S D 8(a=11.38)$ and $q S S D 10(a=14.10)$ produced an epistatic effect of -2.64 , which resulted in an increase of 13.85 spikelets per $10 \mathrm{~cm}$ panicle length in D3. Thus, pyramiding of $q S S D 8$ and qSSD10 also yield denser panicles than S4 (containing qSSD8) and S1 (containing qSSD10). The TSSL T1 carried substitution segments of chromosomes5, 8, and 10. Pyramiding of $q S S D 5, q S S D 8$ and $q S S D 10$ produced an epistatic effect of -9.34 . The genetic effect of the three QTLs in T1 for seed-set density was 21.25 spikelets per $10 \mathrm{~cm}$ panicle length. Thus, pyramiding of $q S S D 5, q S S D 8$, and $q S S D 10$ also yielded denser panicles than S3, S4, and S1. Taken together, all combinations of qSSD5 and qSSD8, qSSD5 and $q S S D 10, q S S D 8$ and $q S S D 10$, and $q S S D 5, q S S D 8$ and $q S S D 10$ resulted in increase in seed-set density (Fig. 4)

The number of secondary branches and spikelets of per panicle of S1, S3, S4, D1, D2, D3, and T1 was significantly higher than that of Nipponbare (Fig. 5E, C). The number of primary branches of S4, D1, D3, and T1 was significantly higher than that of Nipponbare (Fig. 5D). The panicle length of S1, S3, S4, D1, D2, D3, and T1 was significantly lower than that of Nipponbare (Fig. 5B). Thus, the seed-set densities of S1, S3, S4, D1, D2, D3, and T1 were significantly higher than that of Nipponbare (Fig. 5A). In accordance with this finding, $q N P B 8, q N S B 10, q N S B 8, q N S B 5, q S P P 10, q S P P 5$, and $q S P P 8$ all showed additive effects to increase the trait values, whereas $q P L 10, q P L 5$, and $q P L 8$ showed an additive effect to reduce panicle length. The QTLs $q S S D 5, q S S D 8$, and $q S S D 10$ showed additive effects that increased seed-set density.

\section{Genetic analysis of qSSD5, qSSD8, and qSSD10}

For genetic analysis and fine mapping of $q S S D 5, q S S D 8$, and $q S S D 10, \mathrm{~F}_{3}$ populations were constructed by respective recombinant plants. For $q S S D 5$, the chi-square test showed that the numbers of low seed-set density (117) and high seed-set density (383) individuals corresponded to a separation ratio of $1: 3\left(\chi^{2}=\right.$ $\left.0.60<\chi^{2}(0.05,1)=3.84\right)$; for $q S S D 8$, the chi-square test showed that the numbers of low seed-set density (37) and high seed-set density (143) individuals corresponded to the same separation ratio of 1:3 $\left(\chi^{2}=\right.$ $\left.2.86<\chi^{2}{ }_{(0.05,1)}=3.84\right)$; for $q S S D 10$, the chi-square test showed that the numbers of high seed-set density (92) and low seed-set density (308) individuals corresponded to the same separation ratio of 1:3 $\left(\chi^{2}=\right.$ $\left.0.75<\chi^{2}(0.05,1)=3.84\right)$. These results suggest that $q S S D 5$ and $q S S D 8$ showed dominant gene action, whereas $q S S D 10$ showed recessive gene action.

\section{Fine mapping of qSSD 5 and sequence analysis of candidate genes}

Two newly developed SSR markers and 117 recessive individuals with low seed-set density from the $F_{3}$ population were used for fine mapping of qSSD5. The QTL QSSD5 was delimited within a $1.46 \mathrm{Mb}$ interval between the molecular markers RM3170 and SSR1 on chromosome 5 (Fig. 6A). Eight candidate genes for qSSD5 were predicted in this interval. By DNA sequencing of Nipponbare and Z749, candidate gene 1 (auxin-responsive protein), candidate gene 2 (OSIAA19), candidate gene 3 (gibberellin 2-beta- 
dioxygenase), candidate gene 4 (auxin response factor 15), and candidate gene 8 (OsGH3.5; Probable indole-3-acetic acid-amido synthetase) showed no difference between the two lines, which indicates that these five genes were not candidate genes for qSSD5. Candidate gene 5 (OSMPK9), candidate gene 6 (auxin efflux carrier component), and candidate gene 7 (OSMPK17) differed between Nipponbare and Z749. For candidate gene 6 , four SNP differences were detected, of which two were located in the coding sequence (CDS) and caused a change in amino acid, and one each was located in the $5^{\prime}$ untranslated region (UTR) and the 3' UTR (Fig. 6B). For candidate gene 5 (OsMPK9), two SNP differences were detected in the CDS sequence between Nipponbare and Z749 and both caused an amino acid change (Fig. 6C). For candidate gene 7 (OSMPK17), one SNP difference in the CDS sequence was observed between Nipponbare and Z749 and caused an amino acid change from Arginine in Nipponbare to Threonine in Z749 (Fig. 6D). Thus, candidate gene 5 (OSMPK5), candidate gene 6 , and candidate gene 7 (OSMPK17) were potential candidate genes for QSSD5.

\section{Fine mapping of qSSD 8 and sequence analysis of candidate genes}

Twenty pairs of SSR markers were newly developed in the substitution segment of $q S S D 8$, of which two polymorphic markers were detected between Nipponbare and Z749. However, the electrophoresis bands for RM1148 and RM5432 were identical with those of Nipponbare. Thus, the length of the substitution segment of $q S S D 8$ was further shortened from the original $3.06 \mathrm{Mb}$ to $1.53 \mathrm{Mb}$ (Fig. 7A). By candidate gene prediction in the substitution interval, the small auxin-up RNAs OSSAUR31 (LOC_OsO8g02520) and OsSAUR32 (LOC_Os08g02530) were identified as candidate genes for qSSD8. Further sequencing revealed that only OSSAUR32 differed between Nipponbare and Z749. The - 1088th base (located in the promoter region) was mutated from $\mathrm{C}$ in Nipponbare to T in Z749. Thus, OsSAUR32 is a putative candidate gene for $q S S D 8$ (Fig. 7B)

\section{Overlapping substitution mapping of qSSD10}

Given that too few polymorphic markers were detected, fine mapping of $q S S D 10$ was conducted by using overlapping substitution segments of S1 and S2. The substitution segment of S2 was shorter than that of S1. The SSSL S1 carried qSSD10 and showed a seed-set density of 59.97 spikelets per $10 \mathrm{~cm}$ panicle length, which differed significantly from that of Nipponbare (49.75 spikelets per $10 \mathrm{~cm}$ panicle length). In contrast, S2 did not harbor $q S S D 10$ and showed a seed-set density of 50.05 spikelets per $10 \mathrm{~cm}$ panicle length, which did not differ significantly from that of Nipponbare (Fig. 8). Therefore, qSSD10 was subject to further fine-mapping within the substitution interval of RM5348-RM8207-RM3311, with a physical distance of $1.05 \mathrm{Mb}$ (Fig. 8).

\section{Discussion}

\section{The $\mathbf{Z 7 4 9}$ is a useful genetic resource for rice breeding}

To a certain extent, a higher seed-set density is beneficial to increase the grain number and improve grain filling, thereby increasing rice yield. However, seed-set density is a complex trait controlled by multiple 
genes, and is affected by panicle length, number of primary branches, number of secondary branches, and number of spikelets per panicle. Rice CSSLs represent a chromosome segment introgression line library composed of distant genotypes in a superior genetic background, and provide a valuable genetic resource for research into theoretical aspects and the application of complex traits (Furuta et al. 2014). Recently, Li et al. (2019) developed a CSSL population consisting of 75 lines from a cross between XQZB and ZH9308 to identify the QTLs that affect yield-related traits, such as 1000-grain weight, grain length, and grain width. We identified a rice CSSL, Z749, with dense and erect panicles, which carried seven substitution segments from the donor parent Xihui 18 based on the recipient parent Nipponbare. The increased seed-set density of Z749 was caused predominantly by a reduction in panicle length and increases in the numbers of primary and secondary branches, and the number of spikelets per panicle. Z749 harbored many favorable traits useful for rice breeding, in addition to a high seed-set density, including an increased number of spikelets per panicle. Furthermore, no differences in 1000-grain weight or numbers of panicles per plant were observed between Nipponbare and Z749. Therefore, Z749 is a potentially valuable genetic resource for rice breeding.

\section{QTL identification and dissection of complex traits with the Z749, and comparison with previously reported genes}

Given that Z749 carried seven substitution segments from the donor parent Xihui 18 , it was essential to determine the genes distributed in the seven substitution segments and to decompose these segments into different SSSLs. Therefore, we constructed a secondary $\mathrm{F}_{2}$ population by crossing Nipponbare and Z749 to map QTLs for associated traits. We determined that 15 QTLs were distributed on four substitution segments of Z749. The seed-set density of Z749 was controlled by one major QTL ( $q S S D 10$ ) and two minor QTLs ( $q S S D 5$ and $q S S D 8$ ). Compared with the results of previous research, OSPUP7 (27.690-27.691 Mb) was located in the mapping region of $q S P P 5$ and $q S S D 5$. The distance of the linkage marker RM3170 (27.80 Mb) of qSPP5 and qSSD5 from OSPUP7 was $0.11 \mathrm{Mb}$. OsPUP7 encodes a permease with associated purine transport activity involved in cytokinin transport. The pup 7 mutant shows increased plant height, reduced number of spikelets per panicle, and delayed flowering (Qi and Xiong 2013). However, no sequence difference in OsPUP7 was detected between Z749 and Nipponbare, which indicates that OSPUP7 was not the candidate gene for $9 S P P 5$ and $q S S D 5$. The QTLs $q N P B 8$ and qNSB8 were linked with RM1235 (2.12 Mb), and increased the number of primary branches by 0.59 and the number of secondary branches by 3.26 . The gene OSREL2 (3.667-3.675 Mb) was located in this interval, just $1.55 \mathrm{Mb}$ from RM1235. The osre/2 mutant displays a higher number of primary branches but fewer secondary branches compared with those of the wild type (Kwon et al. 2012). However, no difference in the sequence of OSREL2 between Z749 and Nipponbare was detected, which indicates that qNPB8 and $q N S B 8$ were not alleles of OsREL2. The QTL qPL7 might be an allele of OSFAD8 (29.531$29.534 \mathrm{Mb}$ ), given the $0.94 \mathrm{Mb}$ distance of OSFAD8 from RM2715. OsFAD8 encodes a $\omega-3$ fatty acid desaturases. The osfad 8 mutant exhibits decreased plant and panicle length (Nair et al. 2009). Sequencing revealed a difference in the 85th base of the first exon of OSFAD 8 between Nipponbare and Z749, which caused a change in amino acid from Glycine in Nipponbare to Arginine in Z479. The other 
identified QTLs (e.g., qSSD10) have not been studied in any detail. Whether the QTLs correspond to the aforementioned alleles requires verification by further genetic complementation experiments. With regard to whether these QTLs might be cloned alleles, we developed corresponding SSSLs and observed that each line carried many favourable traits useful for breeding (Fig. 5). Consequently, compared with the aforementioned mutants, which often carry adverse traits, the SSSLs can be utilized readily in molecular breeding. In addition, these non-allelic and newly discovered QTLs, such as qSPP5, qSSD5, qSSD10, and qSPP10, can be further fine-mapped and cloned, thereby allowing the underlying molecular mechanism to be investigated in more detail.

\section{SSSLs, DSSLs, and TSSLs are suitable materials for target QTLs analysis}

Single-segment substitution lines are ideal materials for genetic analysis and breeding because they differ in only one substitution segment compared with the recipient parent. Moreover, SSSLs are homozygous and can be used as permanent populations (Zhao et al. 2007). From the results of the primary QTL mapping, we developed five SSSLs (S1 to S5) using a MAS method. These lines were used to validate the stability of 14 QTLs, for which the repeatability of detection was $93.33 \%$. In addition, qNPB7 was also detected by Wang et al. (2020). Thus, these QTLs were genetically stable. In S5 we detected a minor QTL ( $q S P P 7)$, in S3 one QTL ( $q P L 5)$, and in S4 one QTL ( $q P L 8)$, but these QTLs were not detected in the secondary $F_{2}$ segregation population of Nipponbare/Z749 in 2018. These results reveal that the SSSL shows a higher sensitivity of QTL detection, a finding confirmed previously by Zhao et al. (2016b) and Eshed and Zamir (1995). However, one QTL (qNPB10) was not detected repeatedly, which suggests that the detection of some minor QTLs depends on the environment. Liu et al. (2008) highlighted that for a specific environment, the total effect of a QTL includes the main effect and the QTL $\times$ environment $(\mathrm{Q} \times \mathrm{E})$ interaction effect for that environment. In this manner, qNPB10 might only show a QXE interaction effect. Zhao et al. (2016b) observed that different agronomic traits displayed different $\mathrm{Q} \times \mathrm{E}$ interactions and that the Q×E interaction effect is specific to a particular environment. Thus, different SSSLs that differ in the QTLs carried should be treated differently in different applications. Certain SSSLS that carry stable favourable QTLs will be more valuable in molecular breeding.

Chromosome DSSLs and TSSLs combined with corresponding SSSLs can be used to analyse the effect of epistasis interaction between QTLs, which is of importance for genetic analysis of complex traits and application of the target QTL in breeding. We developed three DSSLs (D1 to D3) and one TSSL (T1) using a MAS method, and observed that three SSSLs carried $q S S D 5$ or $q S S D 8$ or $q S S D 10$, which positively affected seed-set density. The interaction between $q S S D 5$ and $q S S D 8, q S S D 5$ and $q S S D 10$, and $q S S D 8$ and qSSD10 all produced negative epistatic effects in DSSLs. However, the sum of the additive and epistasis effects (genetic effect) on seed-set density of each double segment was greater than the value of the additive effect of the largest single QTL. Therefore, DSSLs showed a higher seed-set density compared with that of the single SSSLs. Thus, pyramiding of the QTLs that positively affected seed-set density resulted in a further increase in that trait. We also observed that TSSL T1, which carried qSSD5, qSSD8, and $q S S D 10$, produced a higher seed-set density. These results were important for pyramiding favorable QTLs for yield-related traits. 
Molecular mechanism of rice panicle architecture and analysis of candidate genes for qSSD5, qSSD8, and qSSD10

Elucidation of the molecular mechanism underlying panicle architecture is important for breeding plants with high yield in rice. Considering genes related to development of rice panicles that have been cloned, the majority are associated with plant hormone signalling pathways or MAPK cascades. Auxin can affect plant architecture, and polar auxin transport plays a crucial role in plant growth and development (Zhang and Yuan 2014). Zhao et al. (2015) reported that PAY1 improves plant architecture by affecting polar auxin transport activity and altering endogenous indole-3-acetic acid distribution, and utlimately affects rice panicle architecture and grain number. In the present study, candidate gene 6 (auxin efflux carrier component) for $q S S D 5$ and candidate gene OSSAUR32 for $q S S D 8$ also affected the distribution of auxin. The two genes displayed many SNP differences in sequence between Nipponbare and Z749.

Furthermore, mitogen-activated protein kinase (MAPK) cascades play a crucial role in plant growth and development as well as in biotic and abiotic stress responses (Reyna and Yang 2006). OsMKKK10OsMKK4-OsMPK6 participates in rice panicle morphogenesis, and the GSN1-MAPK module mediates the trade-off between grain number and grain size by integrating localized cell differentiation and proliferation (Guo et al. 2018). OsMPK proteins conteins a highly conserved kinase domain. In the current research, we also observed many SNP differences in OSMPK9 and OSMPK17 between Nipponbare and Z749, the candidate genes for QSSD5. Although OSMPK9 and OSMPK17 have been cloned and OsMPK17 is associated with pathogen infection (Reyna and Yang 2006; Hu et al. 2011), it is important to study further their effects on rice panicle development. Although four candidate genes for $q S S D 5$ and $q S S D 8$ have been identified by gene prediction and DNA sequencing, further functional complementarity verification together with candidate gene analysis of $q S D D 10$ are in progress. This should result in a more complete picture for panicle development for future research.

\section{Conclusions}

Using the cultivar Nipponbare as the genetic background, we identified the rice CSSL Z749, which exhibits a dense and erect panicle phenotype. Z749 carried seven substitution segments derived from Xihui 18 with an average substitution length of $2.16 \mathrm{Mb}$. Fifteen QTLs were distributed on chromosomes 5, 7, 8, and 10 in Z749. The dense panicle of Z749 was caused predominantly by the reduction in panicle length and increases in the numbers of primary and secondary branches and the number of spikelets per panicle. The seed-set density of Z749 is controlled by one major QTL ( $q S S D 10$ ) and two minor QTLs ( $q S S D 5$ and $q S S D 8$ ). The QTLS $q S S D 5, q S S D 8$, and $q S S D 10$ were fine-mapped to intervals of $1.05 \mathrm{Mb}$, $1.46 \mathrm{Mb}$ and $1.53 \mathrm{Mb}$, on chromosomes 10,5 and 8, respectively. Sequencing analysis revealed that two MAPK genes (OSMPKO and OSMPK17) and one gene that encodes an auxin efflux carrier component (candidate gene 6) might be candidate genes for QSSD5, and a small auxin-up RNA (OSSAUR32) might be the candidate gene for GSSD8. Fourteen QTLs were verified by development of five SSSLs, and the repeatability of detection was $93.3 \%$. Epistatic effect analysis revealed that pyramiding of $q S S D 5$ and qSSD8, qSSD5 and $q S S D 10, q S S D 8$ and $q S S D 10$, and $q S S D 5, q S S D 8$, and $q S S D 10$ all produced novel genotypes (D1, D2, D3, and T1) with a higher seed-set density. 


\section{Materials And Methods}

\section{Experimental materials}

The rice CSSL Z749 was developed using Nipponbare as the recipient parent and Xihui 18 as the donor parent. Xihui 18 is an excellent restorer line bred by Southwest University, Chongqing, China. A secondary $F_{2}$ population raised from a cross between Nipponbare and Z749 was used for QTL mapping in 2018. On the basis of the QTL mapping results, nine $F_{2}$ individuals were selected to develop SSSLs, DSSLs and TSSLs by MAS and grown as the lines Z775, Z776, Z777, Z778, Z779, Z780, Z781, Z782 and Z783 in 2019. In addition, three $F_{3}$ segregated populations (Z784-Z786) for fine-mapping of $q S S D 5, q S S D 8$ and qSSD10 were developed from three recombinant plants for $q S S D 5, q S S D 8$, and $q S S D 10$ whose other genetic backgrounds was completely same with that of Nipponbare. Nine homozygous secondary substitution lines in $\mathrm{F}_{4}$ were planted in 2020 for validation of QTLs and epistasis effect analysis.

\section{Field planting}

In 2017, Nipponbare and Z749 were crossed and the seeds were harvested at the experimental station of Southwest University in Chongqing, China. In autumn of the same year, the $F_{1}$ hybrid seeds were sown at the Hainan experimental station and seeds from the $F_{1}$ individuals were harvested. On 10 March 2018, seeds of $Z 749$, Nipponbare, and the $F_{2}$ population comprising 126 individuals were sown at the same experimental station in Chongqing, China. Thirty seedlings of each parent and all $\mathrm{F}_{2}$ seedlings were transplanted to the field on 20 April 2018, with 10 individuals planted per row. The spacing between rows and individual plants was 26.4 and $16.5 \mathrm{~cm}$, respectively. Conventional management practices were applied. In 2019, five $F_{2}$ individuals (Z775, Z776, Z777, Z778, and Z779) for the selection of SSSLs, three $F_{2}$ individuals (Z780, Z781, and Z782) for the selection of DSSLs, one $F_{2}$ individual (Z783) for the selection of TSSLs, together with individuals of Nipponbare were planted. For each material, 30 plants were transplanted. In addition, three segregation population (Z784-786) were planted for fine mapping of $q S S 5, q S S D 8$, and $q S S D 10$, and all plants were transplanted at the same experimental station in Chongqing. In 2020, five SSSLs, three DSSLs, one TSSL, together with Nipponbare and Z749 were planted at the experimental base in Chongqing, again with 30 plants transplanted per line. Development of CSSL$\mathbf{Z 7 4 9}$

First, 263 SSR markers polymorphic between Nipponbare and Xihui 18 were selected from 429 markers that covered the entire rice genome. Repeated backcrossing and selfing in combination with molecular MAS was used from the $\mathrm{BC}_{2} \mathrm{~F}_{1}$ to $\mathrm{BC}_{2} \mathrm{~F}_{7}$ generations derived from Nipponbare as the recipient parent and Xihui18 as the donor parent. In the $\mathrm{BC}_{2} \mathrm{~F}_{7}$ population, a homozygous line with 13 substitution segments designated Z368, which produced dense and erect panicles, was selected. Nipponbare was crossed with Z368 and the progeny were selfed. Twenty individuals from each selfed generation were selected to develop CSSLs by MAS. Finally, CSSL-Z749 with dense and erect panicles, carrying seven substitution segments, was selected in the $F_{3}$ population. The method of substitution segment identification followed 
that of Zhao et al. (2016a). The estimated length of the substitution segment was calculated in accordance with the method of Paterson et al. (1991). MapChart 2.2 software was used to draw the chromosome substitution segment map.

\section{Seed-set density related trait assessment}

At maturity, 10 plants growing on the third to seventh hills of the central two rows of the Nipponbare and $Z 749$ plots, and 126 individuals of the $F_{2}$ populations, were harvested. The panicle length ( $\left.P L\right)$, number of primary branches per panicle (NPB), number of secondary branches per panicle (NSB), number of spikelets per panicle (SPP), number of grains per panicle (GPP) and seed-set density (SSD) were measured. The specific measurement method used for each trait followed Ma et al (2019). The seed-set density was determined as spikelets per $10 \mathrm{~cm}$ panicle length. A student's $t$-test was used to analyse the significance of differences in these traits between Nipponbare and Z749. In addition, descriptive statistics, such as the average, standard deviation, skewness, and kurtosis in the $F_{2}$ population, were calculated using Microsoft Excel 2010.

\section{QTL mapping}

Total genomic DNA of Nipponbare, Xihui $18, Z 749$, and 126 plants from the $F_{2}$ population was extracted using the cetyltrimethyl ammonium bromide method (McCouch et al. 1998). The procedures for PCR amplification, $10 \%$ native polyacrylamide gel electrophoresis, and rapid silver staining were performed in accordance with the methods described by Zhao et al (2016a). Nipponbare bands were scored as " -1 ", Z749 bands were scored as " 1 ", heterozygous bands were scored as " 0 ", and the absence of marker bands was scored as ".". The marker assignments for all markers on the substitution segments of Z749, together with the phenotypic values of each individual in the $F_{2}$ population, were used for QTL mapping. Mapping of QTLs was performed using the restricted maximum likelihood (REML) method implemented in the HPMIXED procedure of SAS statistical software (SAS Institute Inc., Cary, NC, USA) with significance determined at $a=0.05$.

\section{DNA sequence analysis of possible alleles}

Based on the QTLs or genes cloned previously, we speculated whether some of the genes are the causal genes of the QTLs that we mapped. We extracted the genetic information within the 2-Mb QTL mapping interval using Gramene (http://www. gramene.org/rice_mutant/) to predict the candidate gene and found four cloned genes, namely OsFAD8 (Os07g0693800), OsREL2 (Os08g0162100), and OsPUP7 (Os05g0556800), in the corresponding region. We amplified the target gene fragments using genomic DNA of Z749 and Nipponbare as the template using primers for these five genes designed using Vector NTI 10. The PCR products were submitted to Tsingke Biological Technology Co., Ltd (Chongqing, China) for sequencing. The primers used for each gene are listed in Table S1.

\section{Additive and epistatic effect analysis using SSSLs, DSSLs, and TSSLs}


Given that only one substitution segment differed between each SSSL and its recipient parent, each of the SSSLs affecting a quantitative trait carried only a single QTL, and two overlapping SSSLs with a significant effect on the trait carried the same QTL. Identification of the QTL in the SSSL was performed via a Student's $t$-test based on comparison of each SSSL with the recipient parent Nipponbare with significance determined at $a=0.05$. Thus, the QTL controlling a trait that differed from Nipponbare existed on the SSSL when $P<0.05$. The additive effect of the QTL was half the difference between each SSSL and Nipponbare, and the proportional contribution of the additive effect was the additive effect divided by the phenotypic mean for Nipponbare (Zhao et al. 2007). Interaction of QTLs in each DSSL was determined by comparing the difference between (Nipponbare + DSSL) and its corresponding SSSL pairs (SSSLa + SSSLb) at $P<0.05$. The epistatic effect in the DSSL was estimated using half of the mean phenotypic values of [DSSL + Nipponbare) - (SSSLa + SSSLb)]. For the TSSL, a student's $t$-test was first used to detect significant differences between the traits (Nipponbare + Nipponbare + TSSL) and (SSSLa + SSSLb + SSSLc), and when $P<0.05$ it was considered that there was an epistatic effect between these QTLs. The epistatic effect in the TSSL was estimated using half of the mean phenotypic values of [(TSSL + Nipponbare + Nipponbare) - (SSSLa + SSSLb + SSSLc)] (Eshed and Zamir 1996; Zhang et al. 2020).

\section{Fine mapping of $q S S D 5, q S S D 8$, and $q S S D 10$ and candidate gene analysis}

Based on the results of the initial QTL mapping, combined with the MAS selection method, three recombinant plants with only the "0" type band (heterozygous band type) at the $q S S D 5, q S S D 8$, and qSSD10 loci and genetic backgrounds otherwise identical with Nipponbare were selected in the $F_{2}$ generation. In the $\mathrm{F}_{3}$ population, 500 plants for $q S S D 5,180$ plants for $q S S D 8$, and 400 plants for $q S S D 10$ were used for genetic analysis and linkage analysis for fine mapping. The primers for each gene are listed in Table S1. Within the interval for fine mapping of these QTLs, candidate genes were predicted according to annotations in the Gramene (http://www.gramene.org/), Rice Annotation Project (https://rapdb.dna.affrc.go.jp/), and China Rice Data Center (http://www.ricedata.cn/gene/index.htm) databases. Finally, primers for all candidate genes were designed to amplify the target gene fragments using genomic DNA of Z749 and Nipponbare as the template. The PCR products were submitted to Tsingke Biological Technology Co., Ltd (Chongqing, China) for sequencing.

\section{Abbreviations}

QTL: Quantitative trait locus; SSSL: single-segment substitution line; CSSLs: chromosome segment substitution lines; DSSL: double-segment substitution line; TSSL: triple-segment substitution line; SSR: simple sequence repeat; MAS: marker-assisted selection; PL: panicle length; NPB: number of primary branches; NSB: number of secondary branches; SPP: spikelet number per panicle; SSD: seed-set density.

\section{Declarations}

\section{Acknowledgements}


We thank Professor Shizhong Xu at the University of California, Riverside, USA for writing the stem program for the QTL mapping.

\section{Authors' Contributions}

DCW and FMZ conceived and designed the experiments. DCW, KZ, SQX, QLZ, RXL, MML, PXL and FN performed the experiments. DCW, KZ, YHL, GHH, and FMZ analysed the data. DCW and FMZ wrote the paper. All authors read and approved the final manuscript.

\section{Funding}

The study was supported by National Natural Science Foundation of China (32072039) and the Chongqing Technical Innovation and Application Development Project (cstc2019jscx-msxmX0392).

\section{Availability of Data and Materials}

The datasets supporting the conclusions of this article are included within the article.

\section{Ethics Approval and Consent to Participate}

This study complied with the ethical standards of China, where this research work was conducted.

\section{Consent for Publication}

All authors provide their consent for publication.

\section{Competing Interests}

The authors declare that they have no competing interests.

\section{References}

Ashikari M, Sakakibara H, Lin SY, Yamamoto T, Takashi T, Nishimura A, Angeles ER, Qian Q, Kitano H, Matsuoka M (2005) Cytokinin Oxidase Regulates Rice Grain Production. Science 309(5735):741-745.

Balakrishnan D, Surapaneni M, Mesapogu S, Neelamraju S (2019) Development and use of chromosome segment substitution lines as a genetic resource for crop improvement. Theor Appl Genet 132(1):1-25.

Chen J, Gao H, Zheng XM, Jin MN, Weng JF, Ma J, Ren YL, Zhou KN, Wang Q, Wang J, Wang JL, Zhang X, Cheng ZJ, Wu CY, Wang HY, Wan JM (2015) An evolutionarily conserved gene, FUWA, plays a role in determining panicle architecture, grain shape and grain weight in rice. Plant J 83(3):427-438.

Duan PG, Rao YC, Zeng DL, Yang YL, Xu R, Zhang BL, Dong GJ, Qian Q, Li YH (2014) SMALL GRAIN 1, which encodes a mitogen-activated protein kinase kinase 4 , influences grain size in rice. Plant $\mathrm{J}$ 77(4):547-557. 
Eshed Y, Zamir D (1995) An introgression line population of Lycopersicon pennellii in the cultivated tomato enables the identification and fine mapping of yield-associated QTL. Genetics 141(3):1147-1162.

Eshed Y, Zamir D (1996) Less-than-additive epistatic interactions of quantitative trait loci in tomato. Genetics 143(4):1807-1817.

Furuta T, Uehara K, Angeles-Shim RB, Shim J, Ashikari M, Takashi T (2014) Development and evaluation of chromosome segment substitution lines (CSSLs) carrying chromosome segments derived from Oryza rufipogon in the genetic background of Oryza sativa L. Breed Sci 63(5):468-475.

Gathala MK, Ladha JK, Kumar V, Saharawat YS, Kumar V, Sharma PK, Sharma S, Pathak H (2011) Tillage and crop establishment affects sustainability of South Asian rice-wheat system. Agron J 103(4):961971.

Guo T, Chen K, Dong NQ, Shi CL, Ye WW, Gao JP, Shan JX, Lin HX (2018) GRAIN SIZE AND NUMBER 1 negatively regulates the OsMKKK10-OsMKK4-OsMPK6 cascade to coordinate the trade-off between grain number per panicle and grain size in rice. Plant Cell 30(4):871-888.

Guo T, Lu ZQ, Shan JX, Ye WW, Dong NQ, Lin HX (2020) ERECTA1 Acts Upstream of the OsMKKK10OsMKK4-OsMPK6 Cascade to Control Spikelet Number by Regulating Cytokinin Metabolism in Rice. Plant Cell 32(9):2763-2779.

Hu J, Zhou JB, Peng XX, Xu HH, Liu CX, Du B, Yuan HY, Zhu LL, He GC (2011) The Bphio08a gene interacts with the ethylene pathway and transcriptionally regulates MAPK Genes in the response of rice to brown planthopper feeding. Plant Physiol 156(2):856-872.

Huang XZ, Qian Q, Liu ZB, Sun HY, He SY, Luo D, Xia GM, Chu CC, Li JY, Fu XD (2009) Natural variation at the $D E P 1$ locus enhances grain yield in rice. Nat Genet 41(4):494-497.

Keishi K, Masahiko M, Shin U, Yuzuki S, Ikuyo F, Hironobu O, Ko S, Junko K (2003) LAX and SPA: major regulators of shoot branching in rice. Proc Natl Acad Sci USA 100(20):11765-11770.

Kwon Y, Yu SI, Park JH, Li Y, Han JH, Alavilli H, Cho JI, Kim TH, Jeon JS, Lee BH (2012) OsREL2, a rice TOPLESS homolog functions in axillary meristem development in rice inflorescence. Plant Biotechnol Rep 6:213-224.

Li F, Liu WB, Tang JY, Chen JF, Tong HN, Hu B, Li CL, Fang J, Chen MS, Chu CC (2010) Rice DENSE AND ERECT PANICLE 2 is essential for determining panicle outgrowth and elongation. Cell Res 20(7):838-849.

Li M, Tang D, Wang KJ, Wu XR, Lu LL, Yu HX, Gu MH, Yan CJ, Cheng ZK (2011) Mutations in the F-box gene LARGER PANICLE improve the panicle architecture and enhance the grain yield in rice. Plant Biotechnol J 9(9):1002-1013. 
Li SY, Zhao BR, Yuan DY, Duan MJ, Qian Q, Tang L, Wang A, Liu XQ, Zhang J, Wang J, Sun JQ, Liu Z, Feng YQ, Yuan LP, Li CY (2013) Rice zinc finger protein DST enhances grain production through controlling Gn1a/OsCKX2 expression. Proc Natl Acad Sci USA 110(8):3167-3172.

Li Z, Aamir R, Zhang Y, Galal BA, Zhu A, Cao L, Cheng S (2019) Quantitative trait loci for rice yield-related traits using chromosomal segment substitution lines. Rice Sci 26(5):261-264.

Liu GF, Zhang ZM, Zhu HT, Zhao FM, Ding XH, Zeng RZ, Li WT, Zhang GQ (2008) Detection of QTLs with additive effects and additive-by environment interaction effects on panicle number in rice (Oryza sativa L.) with single-segment substitution lines. Theor Appl Genet 116(7):923-931.

Liu GM, Zhang K, Ai J, Deng XJ, Hong YY, Wang XM (2015) Patatin-related phospholipase A, pPLAllla, modulates the longitudinal growth of vegetative tissues and seeds in rice. J Exp Bot 66(21):6945-6955.

Liu Q, Han R, Wu K, Zhang J, Ye Y, Wang S, Chen J, Pan Y, Li Q, Xu X, Zhou J, Tao D, Wu Y, Fu X (2018) Gprotein $\beta Y$ subunits determine grain size through interaction with MADS-domain transcription factors in rice. Nat Commun 9(1):852.

Ma FY, Zhu XY, Wang H, Wang SM, Cui GQ, Zhang T, Yang ZL, He GH, Ling YH, Wang N, Zhao FM (2019) Identification of QTL for kernel number-related traits in a rice chromosome segment substitution line and fine mapping of qSP1. Crop J 7(4):494-503.

McCouch SR, Kochert G, Yu ZH, Wang ZY, Khush GS, Coffman WR, Tanksley SD (1998) Molecular mapping of rice chromosomes. Theor Appl Genet 76:815-829.

Nair PMG, Kang IS, Moon BY, Lee CH (2009) Effects of low temperature stress on rice (Oryza sativa L.) plastid $\omega-3$ desaturase gene, OsFAD 8 and its functional analysis using T-DNA mutants. Plant Cell Tissue and Organ Culture 98(1):87-96.

Paterson AH, Damon S, Hewitt JD, Zamir D, Rabinowitch HD, Lincoln SE, Lander ES, Tanksley SD (1991) Mendelian factors underlying quantitative traits in tomato: comparison across species, generations, and environments. Genetics 127(1):181-197.

Piao RH, Jiang WZ, Ham TH, Choi MS, Qiao YL, Chu SH, Park JH, Woo MO, Jin ZX, An G, Lee J, Koh HJ (2009) Map-based cloning of the ERECT PANICLE 3 gene in rice. Theor Appl Genet 119(8):1497-1506.

Qi ZY, Xiong LZ (2013) Characterization of a Purine Permease Family Gene OsPUP7 Involved in Growth and Development Control in Rice. J Integr Plant Biol 55(11):1119-1135.

Qiao YL, Piao RH, Shi JX, Lee SI, Jiang WZ, Kim BK, Lee J, Han LZ, Ma WB, Koh HJ (2011) Fine mapping and candidate gene analysis of dense and erect panicle $3, D E P 3$, which confers high grain yield in rice (Oryza sativa L.). Theor Appl Genet 122(7):1439-1449. 
Reyna NS, Yang Y (2006) Molecular Analysis of the Rice MAP Kinase Gene Family in Relation to Magnaporthe grisea Infection. Molecular Plant-Microbe Interactions 19(5):530-540.

Shen CJ, Bai YH, Wang SK, Zhang SN, Wu YR, Chen M, Jiang DA, Qi YH (2010) Expression profile of PIN, AUX/LAX and PGP auxin transporter gene families in Sorghumbicolor under phytohormone and abiotic stress. FEBS J 277(14):2954-2969.

Song S, Wang GF, Hu Y, Liu HY, Bai XF, Qin R, Xing YZ (2018) OsMFT1 increases spikelets per panicle and delays heading date in rice by suppressing Ehd1, FZP and SEPALLATA-like genes. J Exp Bot 69(18):4283-4293.

Wang B, Smith SM, Li JY (2018) Genetic Regulation of Shoot Architecture. Annu Rev Plant Biol 69:437468.

Wang H, Zhang JY, Naz F, Li J, Sun SF, He GH, Zhang T, Ling YH, Zhao FM (2020) Identification of rice QTLs for important agronomic traits with long-kernel CSSL-Z741 and three SSSLs. Rice Sci 27(5):414422.

Wu YZ, Fu YC, Zhao SS, Gu P, Zhu ZF, Sun CQ, Tan LB (2016) CLUSTERED PRIMARY BRANCH 1, a new allele of DWARF11, controls panicle architecture and seed size in rice. Plant Biotechnol J 14(1):377-386.

Yoshida A, Ohmori Y, Kitano H, Taguchi-Shiobara F, Hirano HY (2012) ABERRANT SPIKELET AND PANICLE1, encoding a TOPLESS-related transcriptional co-repressor, is involved in the regulation of meristem fate in rice. Plant $\mathrm{J}$ 70(2):327-339.

Zhang B, Shang LG, Ruan B, Zhang AP, Yang SL, Jiang HZ, Liu CL, Hong K, Lin H, Gao ZY, Hu J, Zeng DL, Guo LB, Qian Q (2019) Development of Three Sets of High-Throughput Genotyped Rice Chromosome Segment Substitution Lines and QTL Mapping for Eleven Traits. Rice 12(1):33.

Zhang DB, Yuan Z (2014) Molecular control of grass inflorescence development. Annu Rev Plant Biol 65:553-578.

Zhang T, Wang SM, Sun SF, Zhang Y, Li J, You J, Su T, Chen WB, Ling YH, He GH, Zhao FM (2020) Analysis of QTL for Grain Size in a Rice Chromosome Segment Substitution Line Z1392 with Long Grains and Fine Mapping of $q G L-6$. Rice 13(1):40.

Zhao FM, Tan Y, Zheng LY, Zhou K, He GH, Ling YH, Zhang LH, Xu SZ (2016a) Identification of rice chromosome segment substitution line, Z322-1-10 and mapping QTL for agronomic traits from the $F_{3}$ population. Cereal Res Commun 44(3):370-380.

Zhao FM, Zhu HT, Ding XH, Zeng RZ, Zhang ZM, Li WT, Zhang GQ (2007) Detection of QTLs for Important Agronomic Traits and Analysis of Their Stabilities Using SSSLs in Rice. Agr Sci China 6(7):769-778. 
Zhao FM, Zhu HT, Zeng RZ, Zhang GQ, Xu SZ (2016b) Detection of Additive and Additive $\times$ Environment Interaction Effects of QTLs for Yield Component Traits of Rice using Single Segment Substitution Lines (SSSL). Plant Breeding 135:452-458.

Zhao L, Tan LB, Zhu ZF, Xiao LT, Xie DX, Sun CQ (2015) PAY1 improves plant architecture and enhances grain yield in rice. Plant $\mathrm{J} 83(3): 528-536$.

\section{Figures}

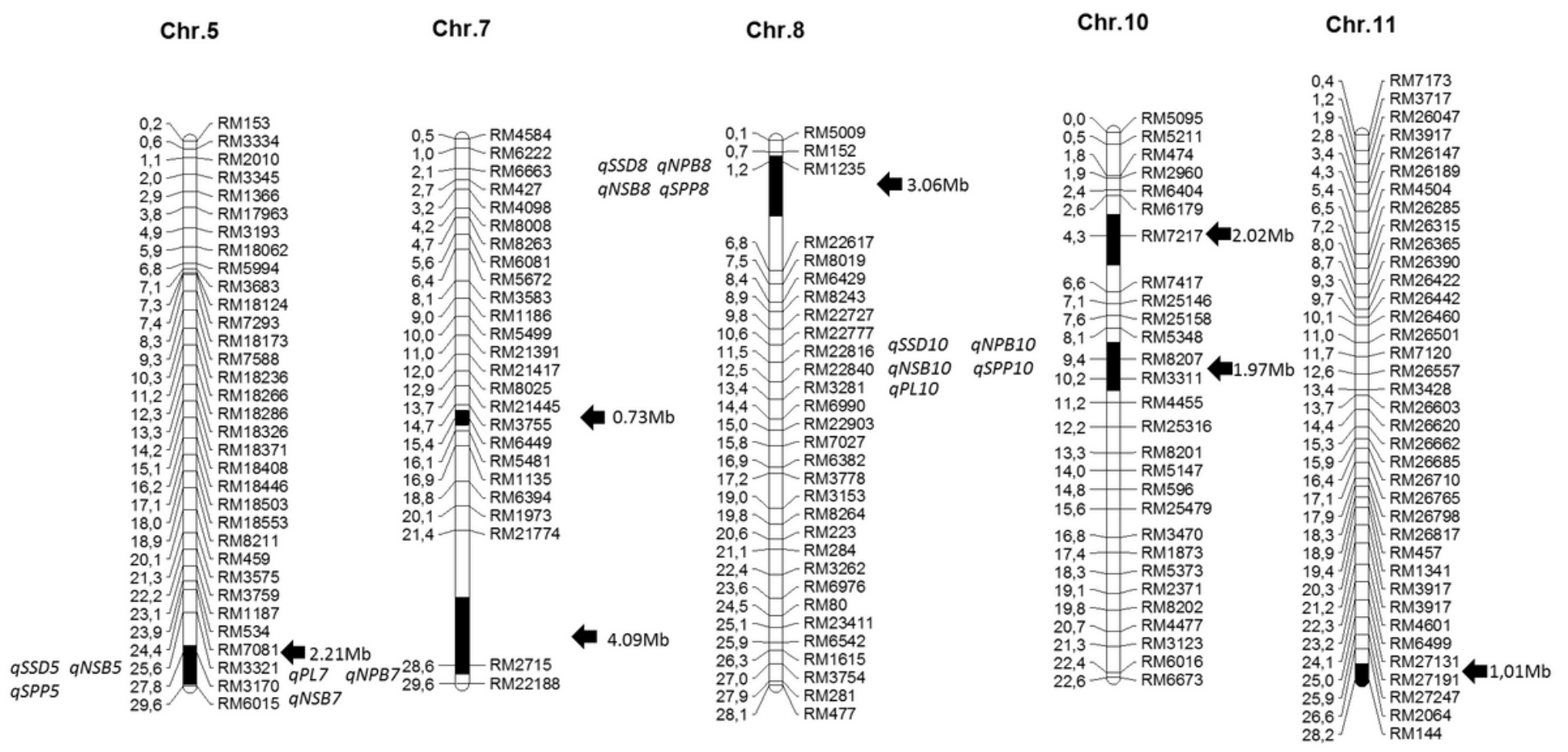

\section{Figure 1}

Substitution segments and detected QTLs in Z749. Physical distances (Mb) and mapped QTLs are marked at the left of each chromosome; markers and substitution length (black arrow direction) are displayed to the right of each chromosome. PL: panicle length, NPB: number of primary branches, NSB: number of secondary branches, SPP: spikelet number per panicle, SSD: seed-set density. 


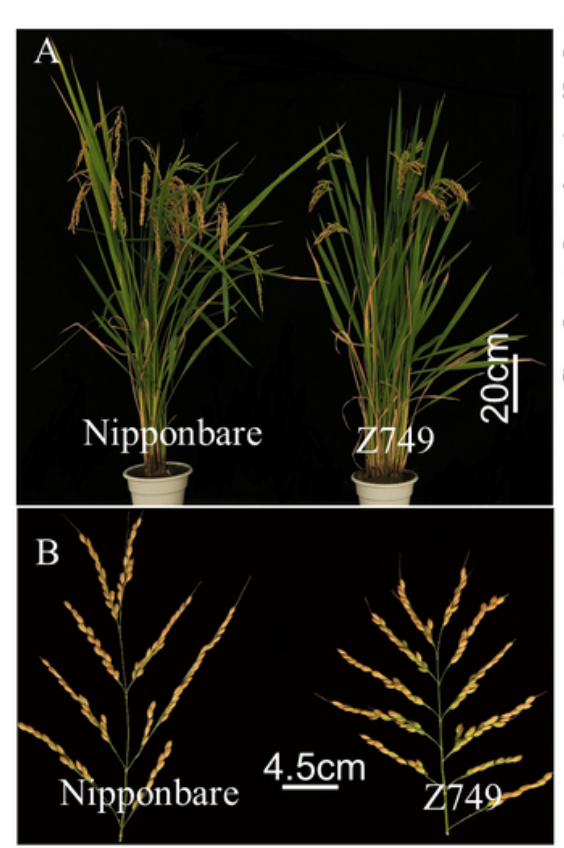

C

D

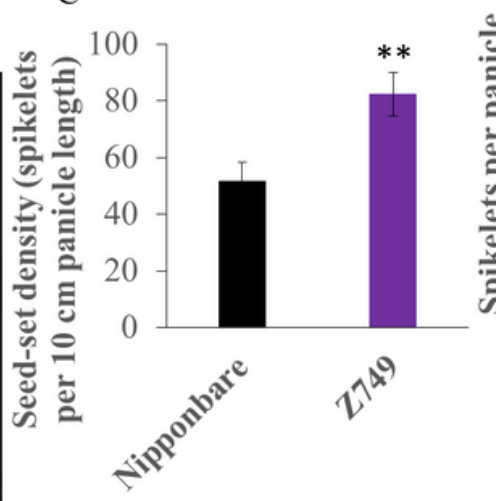

F

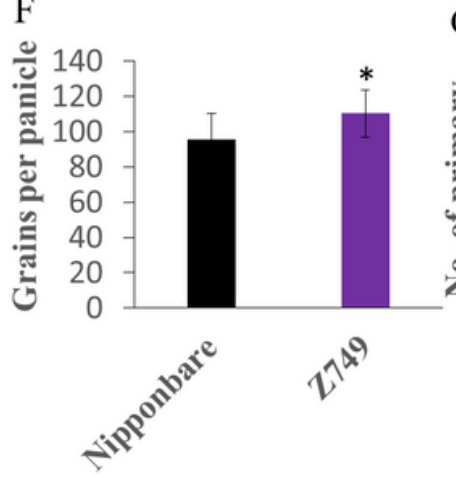

G
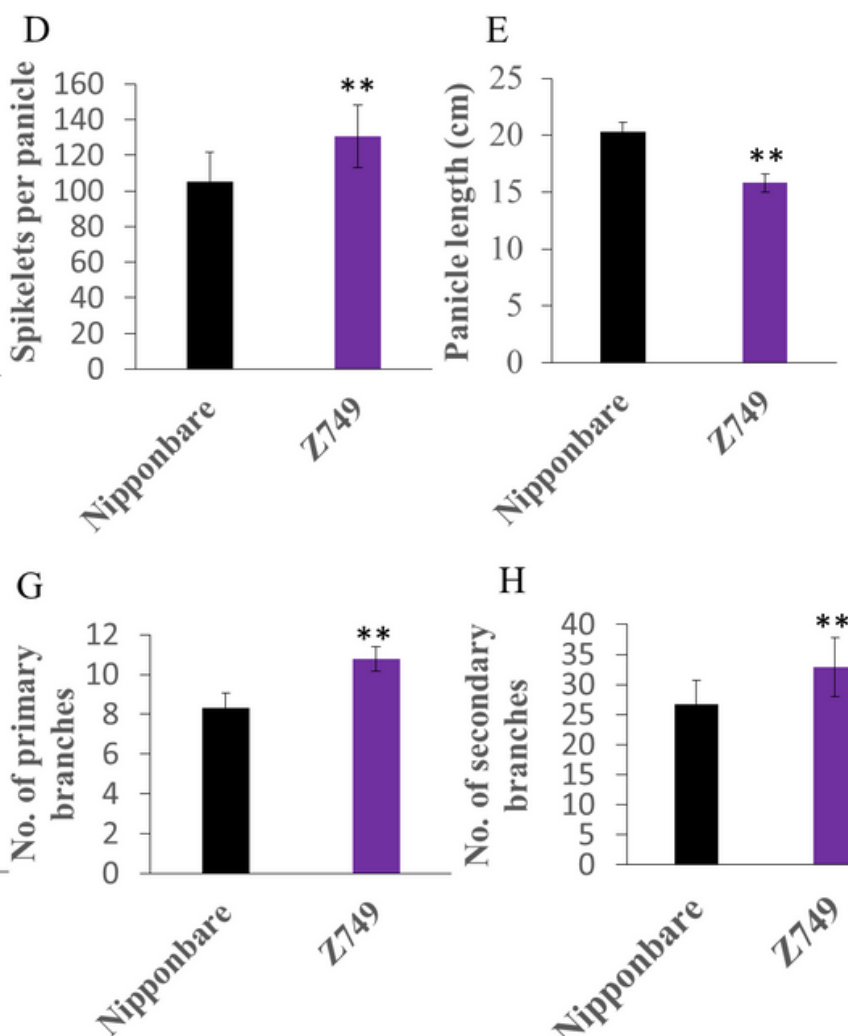

$\mathrm{H}$

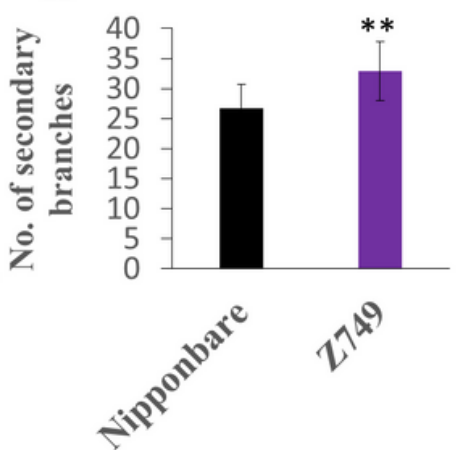

Figure 2

Phenotype of Nipponbare and Z749. A, Plant type of Nipponbare and Z749. B, Panicle of Nipponbare and Z749. C, Seed-set density. D, Spikelet number per panicle. E, Panicle length. F, Grain number per panicle. G, Number of primary branches. $\mathrm{H}$, Number of secondary branches.

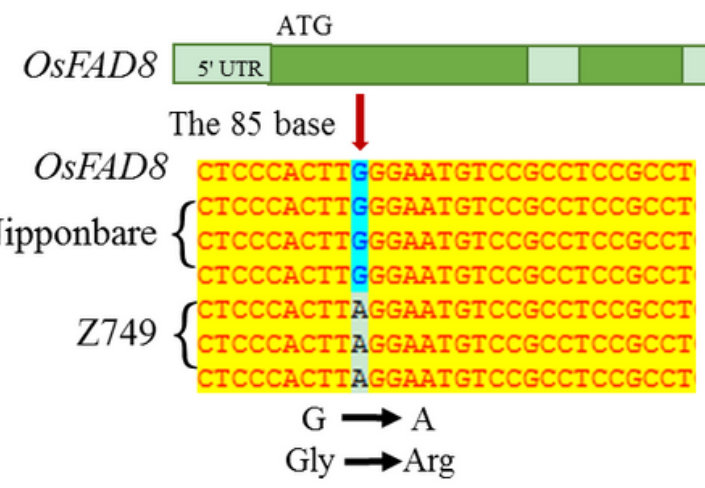

\section{Figure 3}

Blast search of OsFAD8 sequences in Nipponbare and Z749. Sketch map of OsFAD8 (Os07g0693800) gene and difference in sequences of OsFAD8 between Nipponbare and Z749. Green boxes indicate exons, white boxes indicate introns and 3' untranslated region; red arrows indicates the alteration of a codon 


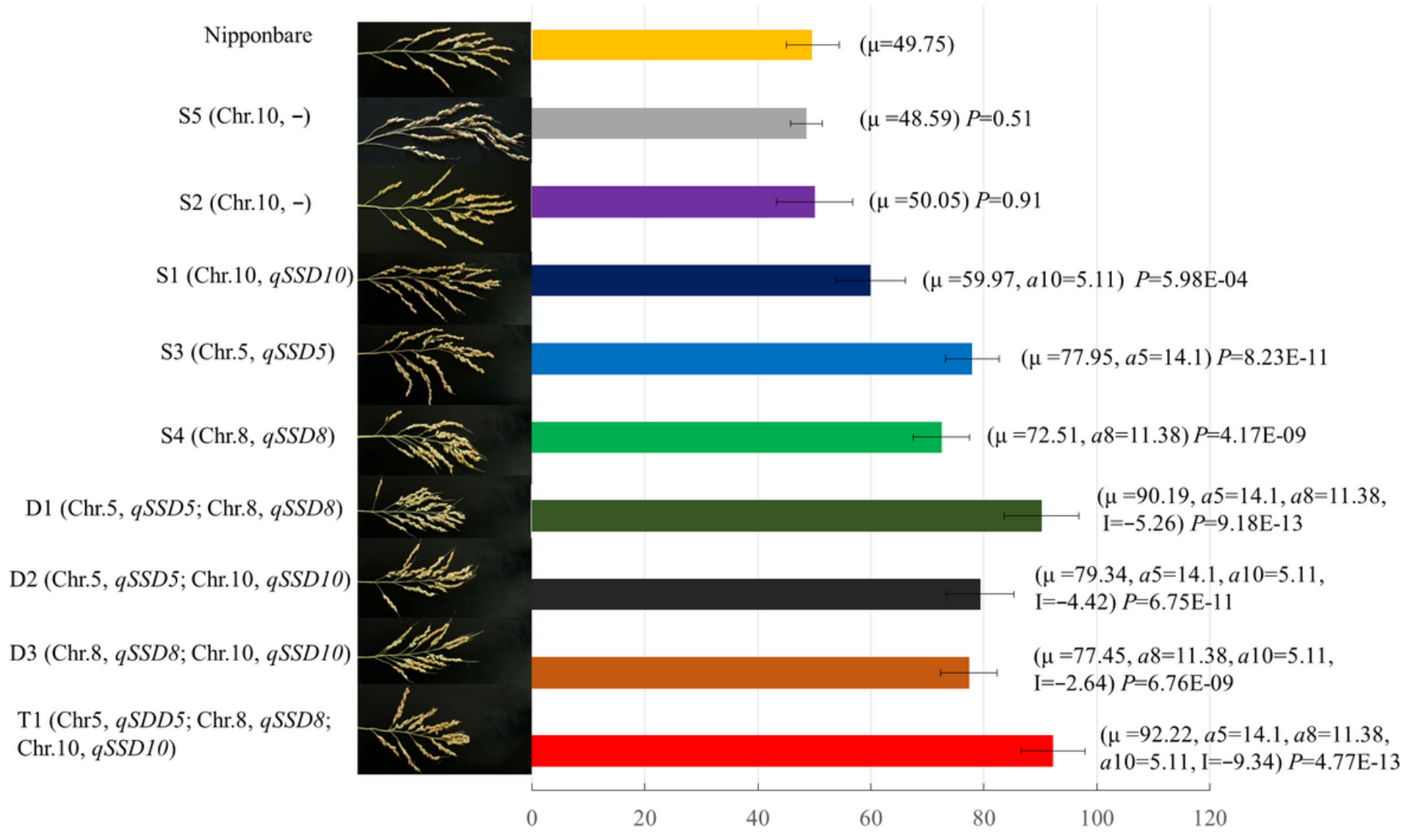

\section{Figure 4}

Phenotype and genotype analysis of nine chromosome segment substitution lines. $\mu$ is the phenotypic value; a denotes the additive effect of the QTL; I denotes the epistatic effect between QTLs. Student's ttest was used to analyse the significance of differences between means. S1: Chr.10, RM5348-RM8207RM3311-RM4455; S2: Chr.10, RM8207-RM3311-RM4455; S3: Chr.5, RM3321-RM3170-RM6015; S4: Chr.8, RM152-RM1235-RM22617; S5: Chr.7, RM21774-RM2715-RM22188. D1: Chr.5, Chr.8, RM3321-RM3170--RM6015, RM152-RM1235-RM22617; D2: Chr.5, Chr.10, RM3321-RM3170-RM6015, RM5348RM8207-RM3311-RM4455; D3: Chr.8, Chr.10, RM152-RM1235--RM22617, RM5348-RM8207-RM3311RM4455; T1: Chr.5, Chr.8, Chr.10, RM3321-RM3170-RM6015, RM152--RM1235-RM22617, RM5348RM8207-RM3311-RM4455. 

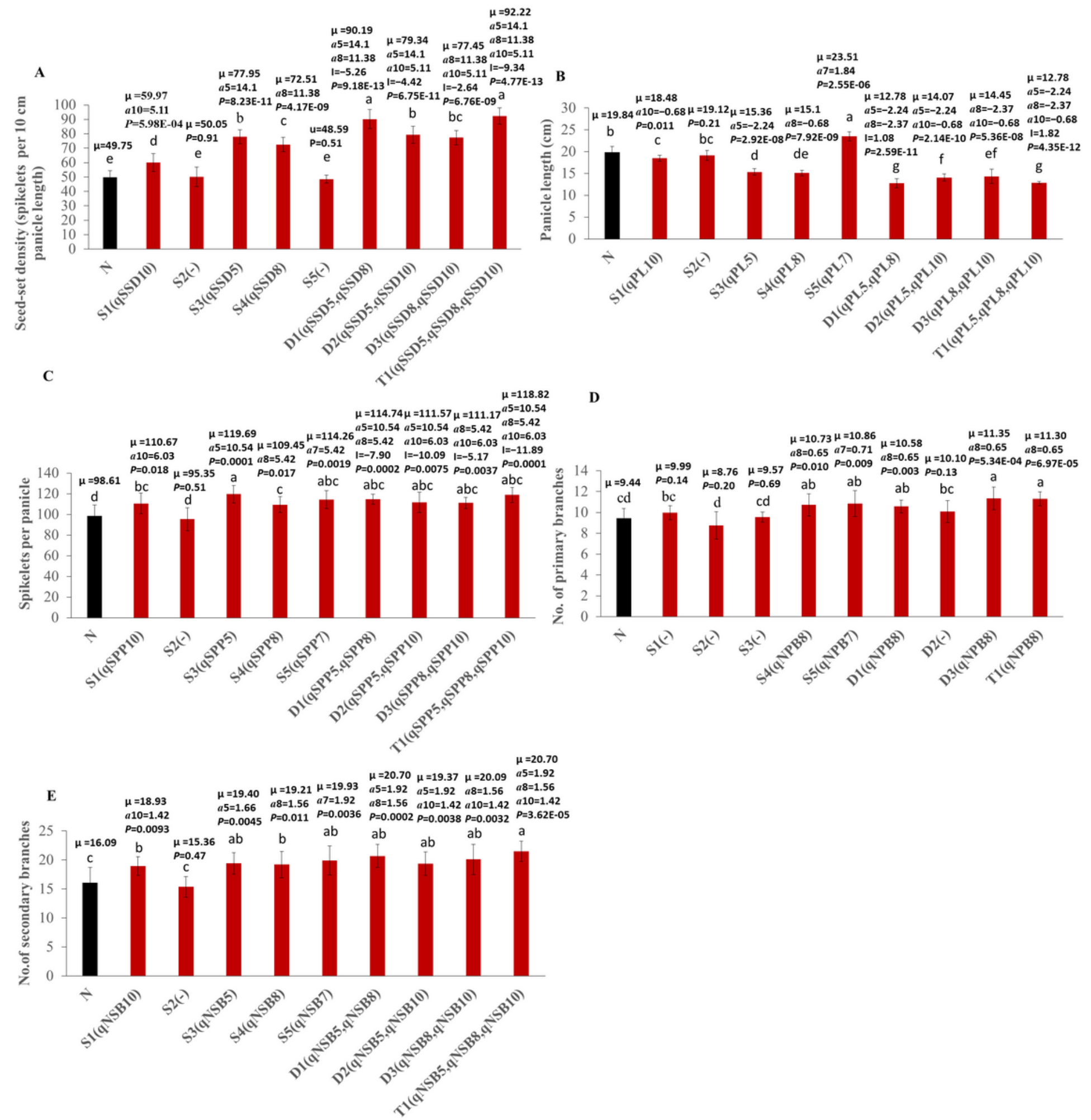

Figure 5

Traits associated with seed-set density of Nipponbare, single-segment substitution lines (SSSLs), doublesegment substitution lines (DSSLs) and triple-segment substitution lines (TSSLs) with the associated quantitative trait loci (QTLs). N: Nipponbare, S: SSSL, D: DSSL, T: TSSL. Different lower-case letters indicate a significant difference $(P<0.05)$ as determined by Duncan's multiple range test. S1: Chr.10, RM5348--RM8207-RM3311--RM4455; S2: Chr.10, RM8207-RM3311--RM4455; S3: Chr.5, RM3321- 
RM3170--RM6015; S4: Chr.8, RM152-RM1235-RM22617; S5: Chr.7, RM21774-RM2715-RM22188. D1: Chr.5, Chr.8, RM3321--RM3170-RM6015, RM152-RM1235--RM22617; D2: Chr.5, Chr.10, RM3321-RM3170--RM6015, RM5348-RM8207-RM3311-RM4455; D3: Chr.8, Chr.10, RM152-RM1235--RM22617, RM5348--RM8207-RM3311--RM4455; T1: Chr.5, Chr.8, Chr.10, RM3321--RM3170--RM6015, RM152RM1235--RM22617, RM5348--RM8207-RM3311-RM4455.

A
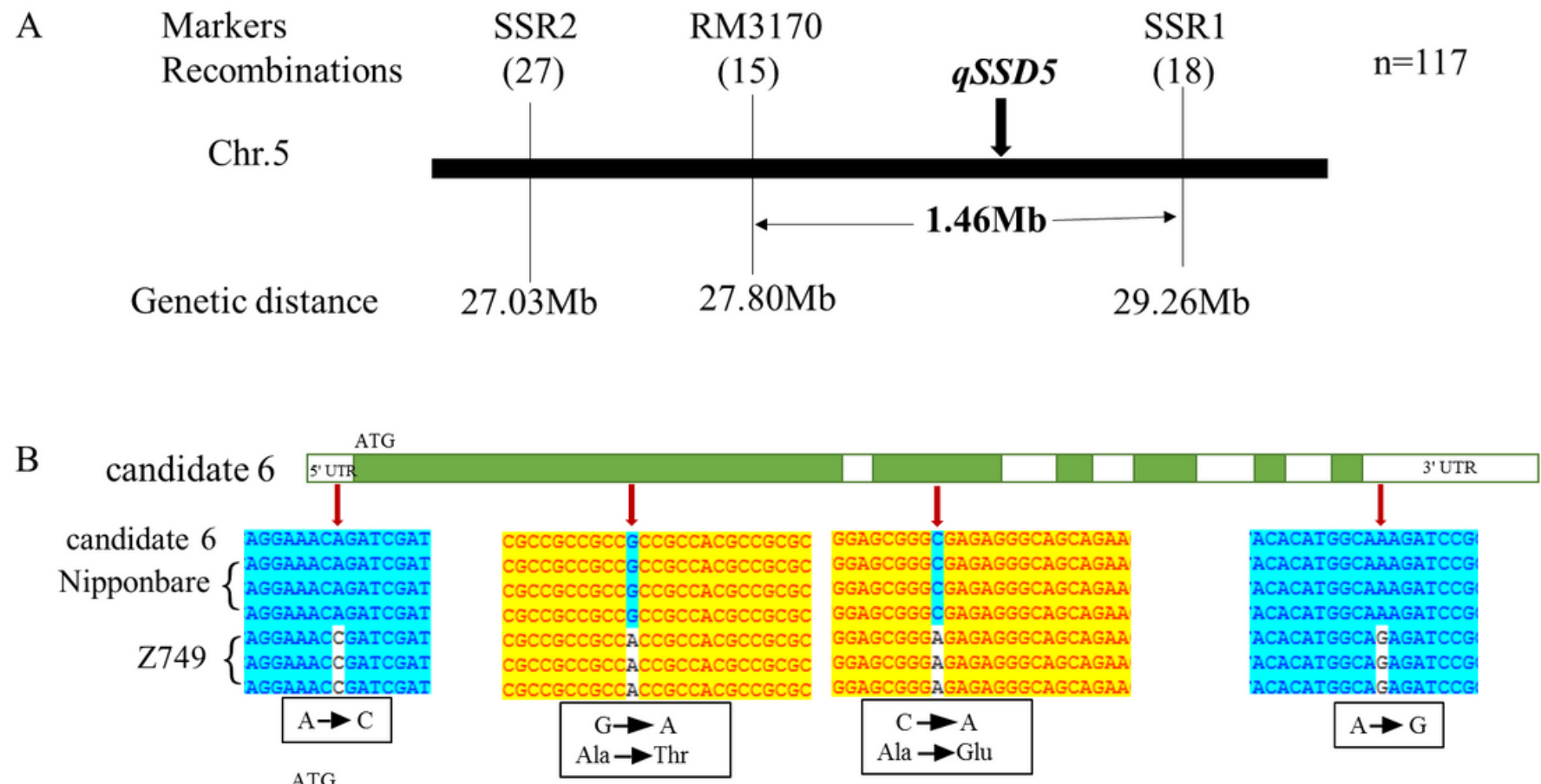

C OsMPK9

\begin{tabular}{|l|l|l|l|l|l|l|}
\hline$T R$ & \\
\hline
\end{tabular}

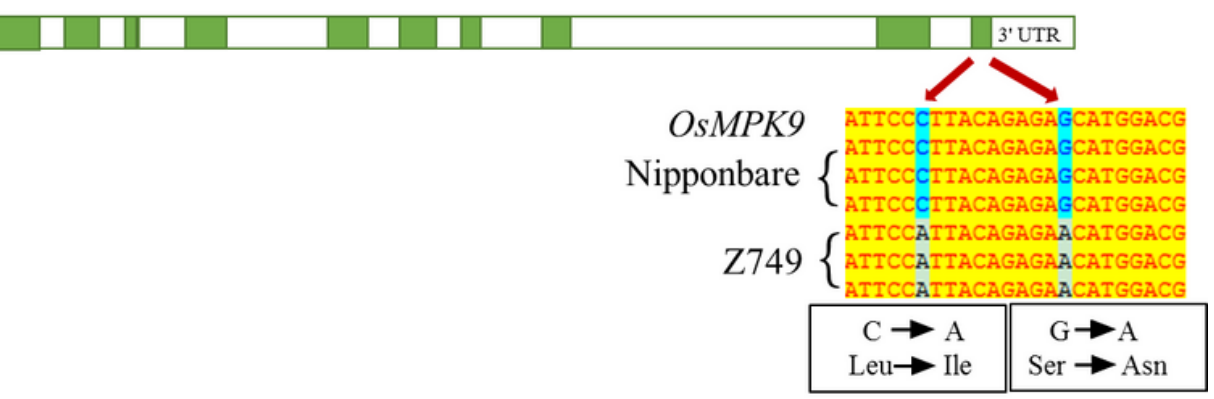

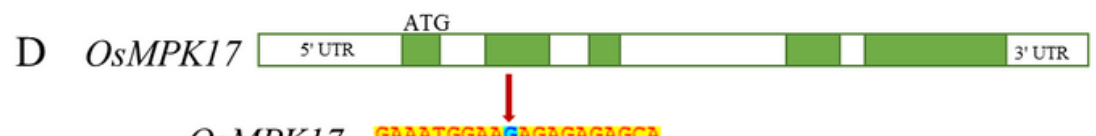

OsMPK17 GAAATGGARGAGAGAGGCA

Nipponbare $\left\{\begin{array}{l}\text { GAAATGGAAGAGAGAGAGCA } \\ \text { GAAATGGAAGAGAGAGACA } \\ \text { GAAATGGAAGAGAGGAGA }\end{array}\right.$

$Z 749\left\{\begin{array}{l}\text { GAAATGGAACAGAGAGAGCA } \\ \text { GAAATGGAACAGAGAGAGCA } \\ \text { GAAATGGAACAGAGAGAGA }\end{array}\right.$

$\begin{aligned} \mathrm{G} & \rightarrow \mathrm{C} \\ \mathrm{Arg} & \rightarrow \mathrm{Thr}\end{aligned}$

\section{Figure 6}

Fine mapping of qSSD 5 and comparative analysis of candidate gene 5 (OsMPK9), candidate gene 6 and candidate gene 7 (OsMPK17). (A) Fine mapping of qSSD5. (B-D) DNA sequences of candidate gene 6, candidate gene 5 (OsMPK9), and candidate gene 7 (OsMPK17) in Z749 compared with those of 
Nipponbare. Green indicates the coding sequence (CDS) of exons and white indicates the introns and untranslated regions (UTR) of the genes. The red arrow indicates the site of a SNP.

A

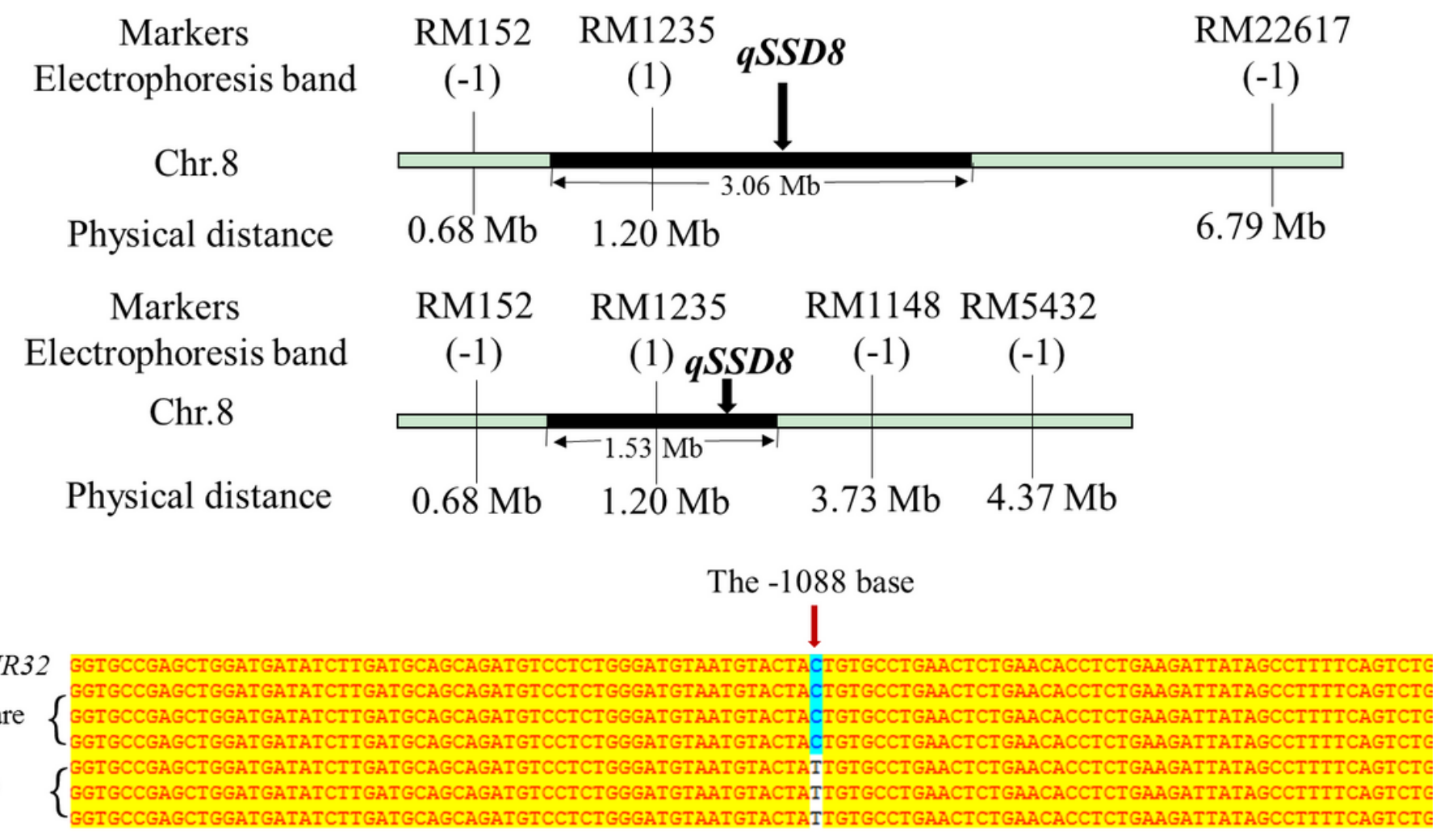

Figure 7

Fine mapping of qSSD8 and sequence analysis of candidate genes. A, qSSD8 was mapped to an interval of $1.53 \mathrm{Mb}$. B, Nucleotide sequence of OsSAUR32 (LOC_Os08g02530) in Z749 compared with that of Nipponbare. -1 indicates that the band for S4 was identical to that of Nipponbare; 1 indicates that the band for S4 differed from that of Nipponbare and was identical with that for Z749 and Xihui18.

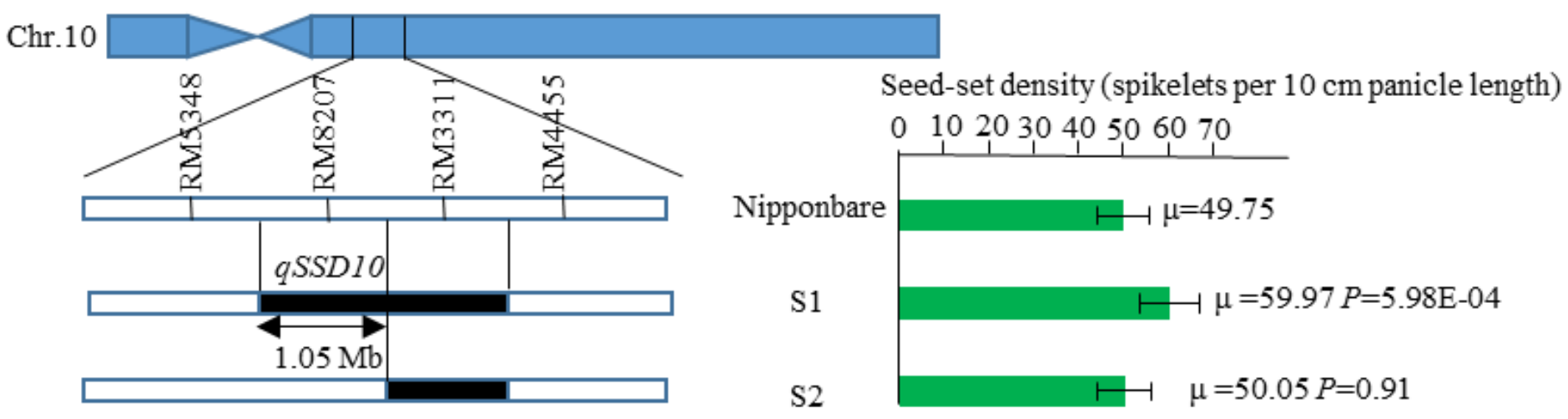

\section{Figure 8}

Fine-mapping of qSSD10. Black regions indicate the substitution segment. S: SSSL. $\mu$ is the phenotypic value. $P$ is the probability value from a Student's t-test for the difference in seed-set density between 
Nipponbare and S1 and S2.

\section{Supplementary Files}

This is a list of supplementary files associated with this preprint. Click to download.

- FiguresandMultipanelfigures.pptx

- TableS1.docx 